\title{
An update on multidrug-resistant tuberculosis 滥
}

\author{
Authors: Mirae Park, ${ }^{A}$ Giovanni Satta ${ }^{B}$ and Onn Min Kon ${ }^{C, D}$
}

Of the 10 million incident cases of tuberculosis (TB) globally in 2017 , around 558,000 cases were rifampicin-resistant of which 82\% were multidrug-resistant (MDR) TB. In England, 5,102 cases were recorded of which 55 cases $(1.8 \%)$ were MDR-TB. MDR-TB cases have worse outcomes and are a serious public health issue.

Polymerase chain reaction (PCR) tests allow a faster approach to diagnose TB and predict drug susceptibility. The emerging use of whole genome sequencing may improve the diagnostic workflow compared with standard drug susceptibility testing, with more rapid molecular sensitivity results and more precise contact investigation of linked cases.

Treatment of MDR-TB remains a challenge as it relies on prolonged second-line drug treatments that are less effective and more toxic than first-line treatments. Two new drug treatments have been approved; bedaquiline and delamanid. In addition, a shorter treatment regimen of 9-12 months can be considered instead of the conventional 20-24 month regimen.

KEYWORDS: Tuberculosis, MDR, multidrug resistant, whole genome sequencing, GeneXpert

\section{Introduction}

Tuberculosis (TB) is an airborne disease caused by Mycobacterium tuberculosis. It affects the lungs, but can also affect other sites causing extrapulmonary TB. Five to fifteen per cent of the estimated 1.7 billion people infected with $M$ tuberculosis will develop TB in their lifetime, with this being higher in patients who are immunosuppressed. ${ }^{1}$ Symptoms of active disease include cough, fever, night sweats and weight loss, and they may be mild for many months, causing further risk of transmission and significant delay in treatment.

Authors: ${ }^{\text {ABRC }}$ clinical research fellow, Department of Respiratory Medicine, Imperial College Healthcare NHS Trust, London, UK; ${ }^{B}$ head of specialty for infection and consultant in infectious diseases and medical microbiology, Imperial College Healthcare NHS Trust, London, UK; ${ }^{C}$ consultant respiratory physician and head of service for tuberculosis, Department of Respiratory Medicine, Imperial College Healthcare NHS Trust, London, UK; ${ }^{D}$ professor of respiratory medicine, National Heart and Lung Institute, Imperial College London, London, UK

\section{Epidemiology}

TB is the ninth leading cause of death worldwide with an estimated 1.6 million deaths from TB in 2017. The epidemiology of TB varies substantially around the globe, with the highest incidence (100 per 100,000 or higher) observed in sub-Saharan Africa, India and south-east Asia. Europe has a low incidence with less than 25 cases per 100,000 . Globally, mortality rates (excluding deaths among HIV-positive people) have decreased by $45 \%$ since 1990 .

In the UK, there have been annual declines in the incidence of TB since 2011. In 2017 the rate of TB was 9.2 per 100,000 (5,102 cases), compared with 9.3 per 100,000 (5,616 cases) in 2016. The main burden of disease was concentrated in large urban areas, with the highest proportion of cases in London (rate of 21.7 cases per 100,000 ), followed by Leicester, Birmingham, Luton, Manchester and Coventry. The number of confirmed or treated as multidrug-resistant (MDR) / rifampicin (RIF)-resistant (RR) TB in the UK has remained stable since 2000 with 61 cases ( 55 cases in England, 3 in Scotland, 3 in Wales and 0 in Northern Ireland) in 2017. ${ }^{2}$

Of the 10 million incident cases of global TB estimated in 2017 , around 558,000 cases were RR-TB of which $82 \%$ were MDR-TB. ${ }^{1}$ MDR-TB is defined as resistance to RIF and isoniazid (INH) with or without resistance to the other first-line drugs. Globally around $3.5 \%$ of MDR-TB/RR-TB were new cases and $18 \%$ were previously treated cases. ${ }^{1}$ India, China and the Russian Federation accounted for $45 \%$ of these cases. ${ }^{1}$ Extensively drug-resistant TB (XDR-TB) is defined as MDR-TB with resistance to any fluoroquinolones and at least one of the second-line injectables - amikacin, capreomycin or kanamycin. It accounts for $7.4 \%$ of MDR-TB worldwide. ${ }^{1}$

\section{Diagnostic problems in TB}

Globally there still remains a large gap (64\%) between the incidence and number of new TB cases diagnosed. Only 2 million of the 6.6 million (30\%) notified cases were tested for RIF resistance, $24 \%$ for new and $70 \%$ for previously treated TB patients. $^{1}$

Culture-confirmed TB has been reported in England at $61.8 \%$ in 2017, which has slightly decreased from $64 \%$ in 2016 but similar to previous years. Sixty-three per cent of pulmonary TB patients had sputum smear results reported and $56 \%$ were positive. ${ }^{2}$ With the goals of the World Health Organization (WHO) End TB Strategy which aims to achieve a $90 \%$ reduction in TB deaths and $80 \%$ reduction in TB incidence by $2030,{ }^{1}$ there has been much focus on the development of diagnostic tests, including point of care tests.

For MDR-TB, rapid molecular diagnostic tests such as the Xpert MTB/RIF, a cartridge-based automated molecular assay which was 
endorsed by WHO in 2016 as an initial test for TB and detection of RIF resistance, has significantly increased case detection and improved RIF resistance identification from sputum. ${ }^{3}$ It is a polymerase chain reaction (PCR) test which requires limited technical expertise and is able to produce a result within 2 hours. The conventional smear microscopy results can take up to 2 days and have a variable sensitivity from $32-94 \% .{ }^{4}$ Conventional culture methods can take up to 8 weeks for the final report. ${ }^{5}$ Firstline drug susceptibility testing (DST) takes a further 2-3 weeks, but if there is evidence of resistance then a second-line DST is undertaken which takes a further 2-3 weeks, introducing more delay before a full DST profile is available for resistance cases and meaning a possible delay in appropriate treatment of MDR-TB.

Some studies have shown that the sensitivity for Xpert MTB/ RIF is inadequate in patients with paucibacillary disease hence limiting its use in smear-negative and extrapulmonary disease. This is particularly relevant in HIV patients and for children. ${ }^{6-8}$ The Xpert MTB/RIF Ultra has subsequently been developed to try to overcome these limitations with two multi-copy amplification targets (IS6110 and IS1081) and improved sensitivity. ${ }^{9}$ A recent multicentre prospective study by Dorman et a ${ }^{10}$ has compared the diagnostic accuracy of Xpert MTB/RIF with the Xpert MTB/RIF Ultra in sputum samples. It highlighted that Xpert MTB/RIF Ultra was superior to Xpert MTB/RIF in patients with paucibacillary disease and in patients with HIV. The increased sensitivity came at the expense of slightly decreased specificity. However, Xpert MTB/RIF Ultra and Xpert MTB/RIF performed similarly in detecting RIF resistance. ${ }^{10}$

Other point-of-care diagnostics which WHO have also endorsed are loop-mediated isothermal amplification (TB-LAMP) and the lateral flow lipoarabinomannan (LAM) assay. All point-of-care diagnostic tests have their strengths as well as limitations, but the aim is to have rapid diagnostic tests which are affordable at decentralised settings with minimal expertise required. ${ }^{11}$

Another recent development in MDR-TB diagnostics is the emergence of whole-genome sequencing (WGS). Unlike targeted molecular tests which only examine a limited number of target regions, WGS provides a rapid comprehensive view of the genotype of $M$ tuberculosis and those single nucleotide polymorphisms (SNPs) responsible for or associated with resistance. This can be used to predict drug resistance. ${ }^{12}$ WGS is also useful in reviewing transmission events in outbreak situations by determining genetic relatedness. Up until recently, the mycobacterial interspersed repetitive unit-variable number of tandem repeats (MIRU-VNTR) method was used to determine outbreaks in the UK. This was able to suggest clusters of isolates, but unlike WGS lacked the accuracy to be certain of linked transmissions. ${ }^{12}$ WGS provides more information than MIRUVNTR strain typing on how isolates are related to each other, and whether isolates are likely to be part of the same transmission chain and timing and direction of transmission, and hence plays a significant role in public health intervention. ${ }^{2}$

In 2017, England became the first country in the world to introduce and pioneer the use of WGS on a national scale for the diagnosis, detection of drug resistance and typing of $M$ tuberculosis, and other countries may soon follow (Netherlands and Norway). ${ }^{13}$ With these advances in diagnostic techniques, the workflow is improving, especially compared with the standard DST, with results being available faster that potentially improve clinical outcome and establishing patients on the correct treatment regime at the earliest timeframe.

\section{Treatment for MDR-TB}

Effective TB chemotherapy began in 1952 and since then strains of $M$ tuberculosis have acquired resistance to various drugs compromising both treatment and control programmes. MDR and XDR-TB have emerged from inappropriate use of anti-TB medications, incorrect prescriptions, poor quality drugs and ending treatment prematurely. ${ }^{13}$

Surveys carried out by WHO in countries with a high TB or MDRTB burden show a slight increase in the proportion of MDR-TB in all TB cases with the burden of MDR-TB increasing faster or decreasing slower compared with the overall TB burden. Globally only $25 \%$ of the estimated MDR/RR-TB incidence cases were started on second-line MDR-TB treatment. ${ }^{1}$ Notably only $55 \%$ of MDR-TB and $34 \%$ of XDR-TB are successfully treated.

A retrospective study in the UK looking at 100 consecutive cases of MDR-TB outcomes and treatment between 2008 and 2014 highlighted some of the complex issues associated with MDR-TB. The results showed that median hospital admission was 62.5 days; longer admission was associated with pulmonary cases, cavities on chest radiograph and a public health policy of waiting for sputum culture conversion. Notably, $13 \%$ lost their housing. The median time for sputum culture conversion was 33.5 days. Treatment outcome was high at $74 \%$ and mortality was low at $1 \%$. Fourteen per cent had negative outcomes with poor adherence being the commonest cause. ${ }^{15}$

Conventional treatment for MDR-TB is challenging as it requires at least five anti-TB drugs for at least 20 months and can cause significant adverse effects. The selection of drugs should be based on previous history, patterns of resistance and DST data if available. $^{16}$

For the first time in more than 50 years, two new anti-TB treatments have been approved; bedaquiline and delamanid. Bedaquiline is a diarylquinoline (TMC207) which inhibits bacterial ATP synthase, and acts on drug-sensitive and resistant $M$ tuberculosis, killing both replicating and non-replicating bacilli. The addition of bedaquiline to standard therapy for MDR-TB has been shown to reduce the time to sputum culture conversion with the rates of conversion to a negative sputum culture at $48 \%$ with bedaquiline and $9 \%$ with placebo at 8 weeks. ${ }^{17}$ Further studies have shown that bedaquiline reduces the median time to culture conversion compared with placebo from 125 to 83 days, and at 24 weeks, and increases the culture conversion rate to $79 \%$ from $58 \%$. The cure rates at 120 weeks were $58 \%$ compared with $32 \%$ in placebo. ${ }^{18}$

Delamanid is the first treatment in the novel nitro-dihydroimidazooxazole class that inhibits mycolic acid synthesis. In a randomised controlled multinational trial with 481 patients, delamanid was associated with increased sputum culture conversion at 2 months. Thirteen per cent of patients receiving the higher dose of $200 \mathrm{mg}$ had a prolonged QT interval compared with $3.8 \%$ in placebo, but this was not associated with clinical events such as syncope. ${ }^{19}$ A further observational study showed that delamanid improved the outcomes and reduced the mortality to $1 \%$ from $8.3 \%$ with placebo in patients with MDR-TB. Treatment benefits were also seen in XDR-TB. ${ }^{20,21}$

WHO has been publishing guidelines for drug-resistant TB since 1997 and the most recent full update was in 2016. It updated classifications for second-line drugs based on current evidence and safety, and has incorporated the new anti-TB drugs using groups $A$ to $D$ (see Table 1). ${ }^{22}$ This is to help construct a drug regime 
Table 1. World Health Organization guidelines for drug-resistant tuberculosis, 2016 update $^{a}$

Group

A. Fluroquinolones ${ }^{b}$

B. Second-line injectable agents

C. Other core second-line agents ${ }^{b}$

D. Add-on agents (not part of the core MDR-TB regimen)

\section{Medicine}

Levofloxacin

Moxifloxacin

Gatifloxacin

Amikacin

Capreomycin

Kanamycin

(Streptomycin) $^{c}$

Ethianomide / Prothionamide

Cycloserine / Terizidone

Linezolid

Clofazimine

D1 Pyrazinamide

Ethambutol

High-dose isoniazid

D2 Bedaquiline

Delamanid

D3

p-aminosalicylic acid

Imipenem-cilastatin $^{d}$

Meropenem $^{d}$

Amoxicillin-clavulanate $^{d}$

(Thioacetazone) ${ }^{\mathrm{e}}$
Abbreviation

Lfx

Mfx

Gfx

Am

$\mathrm{Cm}$

$\mathrm{Km}$

(S)

Eto / Pro

Cs / Trd

Lzd

$\mathrm{Cfz}$

Z

$\mathrm{H}$

Bdq

DIm

PAS

Ipm

$\mathrm{Mpm}$

$\mathrm{AMx}-\mathrm{Clv}$

(T)

MDR-TB = multidrug-resistant tuberculosis

${ }^{a}$ This regrouping was intended to guide the design of longer regimens; the composition of the recommended shorter MDR-TB regimen is standardised.

${ }^{b}$ Medicines in Groups $\mathrm{A}$ and $\mathrm{C}$ are shown by decreasing order of usual preference for use (subject to other considerations).

${ }^{C}$ Refer to the source text for the conditions under which streptomycin may substitute other injectable agents. Resistance to streptomycin alone does not qualify for the definition of extensively drug-resistant tuberculosis.

${ }^{d}$ Carbapenems and clavulanate are meant to be used together; clavulanate is only available in formulations combined with amoxicillin.

eHIV status must be confirmed to be negative before thioacetazone is started.

Reprinted with permission from World Health Organization. WHO treatment guidelines for drug-resistant tuberculosis, 2016 update. Geneva: WHO, 2016:23. ${ }^{22}$

consisting of at least four active drugs. ${ }^{23}$ Group A includes the later generation fluoroquinolones, group B includes injectables, group C includes other core second-line drugs, and group D comprises add-on drugs that are used in drug resistance or intolerance. Delamanid or bedaquiline can be added if there is quinolone or injectable resistance. Para-aminosalicylic acid and carbapenems with clavulanate are also reserve drugs. ${ }^{23}$

The longer conventional MDR-TB regimen should consist of at least five effective TB drugs during intensive phase (up to 8 months) including pyrazinamide, and four core second-line TB medications, one from group $A$, one from group $B$ and at least two from group $C$. If the minimum of effective TB medicines cannot be composed, an agent from group D2 and other agents from D3 may be added to bring the total to five. It is also recommended that this regimen can be further strengthened by high-dose isoniazid and or ethambutol. The continuous phase is usually for 12 months or more and composed of three or more second-line drugs. ${ }^{22}$

In 2016, WHO approved the use of a 'short' standardised regimen for MDR-TB or RR-TB in patients who do not have strains that are resistant to fluoroquinolones or second-line injectables, shortening the duration of treatment to $9-12$ months. ${ }^{22}$ This is only recommended under specific conditions and is still a conditional recommendation. ${ }^{22}$ There have been numerous studies supporting this shortened regimen such as the 'Bangladesh regimen' which used high-dose gatifloxacin, clofazimine, ethambutol and pyrazinamide supplemented by prothionamide, kanamycin and double-dose isoniazid during the 4 month intense phase, which cured $87.9 \%$ of the patients with no relapse. ${ }^{24} \mathrm{WHO}$ recommended a $4-6$ month intensive phase substituting moxifloxacin for gatifloxacin. The intensive phase was followed by a 5 month continuation phase of moxifloxacin, clofazimine, ethambutol and pyrazinamide. Together with the STREAM study, a multicentre randomised trial comparing the WHO standard MDR-TB regimen (20-24 months) with the current MDR-TB regimen ( 9 months), should provide further evidence to guide MDR-TB treatment. At the interim stage, the short regimen is non-inferior and with similar adverse event rates. Stage two of the trial is assessing bedaquiline as part of the shorter regimen. ${ }^{25}$

A recent systematic review and meta-analysis by Bastos et al that included 74 studies highlighted the higher treatment success for MDR-TB with individualised regimens 
Table 2. Proposed 2018 grouping of medicines recommended for use in longer multidrug-resistant tuberculosis regimens

\begin{tabular}{|c|c|c|}
\hline Group & Medicine & Abbreviation \\
\hline \multirow[t]{3}{*}{ Group A: Includes all three medications (unless they cannot be used) } & Levofloxacin or moxifloxacin & Lfx or Mfx \\
\hline & Bedaquiline $^{a, d}$ & Bdq \\
\hline & Linezolid $^{b}$ & Lzd \\
\hline \multirow[t]{2}{*}{ Group B: Add both medicines (unless they cannot be used) } & Clofazimine & $\mathrm{Cfz}$ \\
\hline & Cycloserine or terizidone & Cs or Trd \\
\hline \multirow{7}{*}{$\begin{array}{l}\text { Group C: Add to complete the regimen and when medicines from } \\
\text { Group A and B cannot be used }\end{array}$} & Ethambutol & $\mathrm{E}$ \\
\hline & Delamanid ${ }^{c, d}$ & DIm \\
\hline & Pyrazinamide $e^{e}$ & Z \\
\hline & Imipenem-cilastatin or Meropenem ${ }^{f}$ & Ipm-Cln or Mpm \\
\hline & Amikacin (or streptomycin) ${ }^{g}$ & Am or $\mathrm{S}$ \\
\hline & Ethionamide or prothionamide & Eto or Pto \\
\hline & P-aminosalicylic acid & PAS \\
\hline \multicolumn{3}{|c|}{$\begin{array}{l}\text { 'Evidence on the safety and effectiveness of Bdq beyond } 6 \text { months was insufficient for review; extended Bdq use in individual patients will need to follow 'off-label' } \\
\text { use best practices. } \\
\text { bOptional duration of use of Lzd is not established. Use for at least } 6 \text { months was shown to be highly effective, although toxicity may limit its use. } \\
\text { 'The position of DIm will be re-assessed once individual patient data from trial } 213 \text { has been reviewed; these data were not available for the evidence assessment } \\
\text { in July outlined above. Evidence on the safety and effectiveness of DIm beyond } 6 \text { months was insufficient for review; extended use of DIm in individual patients will } \\
\text { need to follow 'off-label' use best practices. } \\
\text { dEvidence on concurrent use of Bdq and Dlm was insufficient for review. } \\
\text { eZ is only counted as an effective agent when DST results confirm susceptibility. } \\
\text { fAmoxicillin-clavulanic acid is administered with every dose of Imp-Cln or Mpm but is not counted as a separate agent and should not be used as a separate agent. } \\
{ }^{9} \text { Am and S are only to be considered if DST results confirm susceptibility and high-quality audiology monitoring for hearing loss can be ensured. S is to be considered only } \\
\text { if Am cannot be used and if DST results confirm susceptibility (S resistance is not detectable with second-line molecular line probe assays and phenotypic DST is required). } \\
\text { Reprinted with permission from World Health Organization. Rapid Communication: Key changes to treatment of multidrug-and rifampicin-resistant tuberculosis } \\
\text { (MDR/RR-TB). Geneva: WHO, 2018:3-4. }\end{array}$} \\
\hline
\end{tabular}

compared with standardised treatments (64\% vs $53 \%$ ). Pooled treatment success was $60 \%$ in patients with MDR-TB, and $26 \%$ in patients with XDR-TB. One of the key challenges and important aspects of MDR-TB case management is monitoring drug-related side effects. According to the results of the DOTS-Plus initiative, the most common side effects were nausea and vomiting (32.8\%) and diarrhoea $(21 \%)$. There was a $12 \%$ incidence of hearing disturbance. ${ }^{26}$ Seventy-nine per cent of patients experienced at least one adverse event with a median of three adverse events per patient. Twenty per cent of the cases led to a change in the drug dose and $64 \%$ of the cases had at least one drug temporarily or permanently discontinued. ${ }^{27}$

There are currently 14 new TB drugs are in the pipeline. Three new drugs for MDR-TB are in advanced stages of development and nine are being assessed in phase 1-2 trials. These include bedaquiline, delamanid and pretomanid in phase 3 , and newer oxazolidinones such as sutezoid and delpazolid, with DprE1 inhibitors in phase 1 and 2. There are also immune-based treatments and host-directed therapies under development with the view to eliminating $M$ tuberculosis, shortening treatment duration and preventing new drug resistance. ${ }^{28}$

There has also been a move towards injectable-free regimens to reduce the significant toxic effects (ototoxicity and nephrotoxicity) and the logistics of IV administration, and to improve treatment compliance. The NeXT trial is currently looking into all oral medications of bedaquiline, linezolid, levofloxacin and pyrazinamide for 6-9 months in the treatment of MDR-TB. In keeping with this and recent meta-analyses of retrospective data $^{29}$ at the time of publication, WHO have released an initial communication revising the grouping of TB medications from $\mathrm{A}$ to $\mathrm{C}$ for longer MDR-TB regimens (Table 2). ${ }^{30}$ This major revision has relegated the use of amikacin as a reserve drug in group $C$ with bedaquiline now moved to group $\mathrm{A}$.

\section{Conclusion}

MDR-TB remains a global challenge and focus needs to be on preventing the development of drug resistance through appropriate treatment of drug-susceptible TB. Alongside improved access to novel and effective treatments with fewer adverse effects, the ongoing development of rapid diagnostic approaches to detect TB and reliably predict drug resistance will help achieve the goal of ultimately eliminating TB.

\section{References}

1 World Health Organization. Global tuberculosis report 2018. Geneva: WHO, 2018. www.who.int/tb/publications/global_report/en/ [Accessed 19 September 2018].

2 Public Health England. TB in England: 2018 - Presenting data to end of 2017. London: PHE, 2018. https://assets.publishing.service.gov.uk/ government/uploads/system/uploads/attachment_data/file/742782/ TB_Annual_Report_2018.pdf [Accessed 05 October 2018].

3 World Health Organization. Xpert MTB/RIF assay for the diagnosis of pulmonary and extrapulmonary TB in adults and children. automated real-time nucleic acid amplification technology for rapid and simultaneous detection of tuberculosis and rifampicin resistance: Xpert MTB/ RIF assay for the diagnosis of pulmonary and extrapulmonary TB in adults and children: policy update. Geneva: WHO, 2013. www.ncbi. nlm.nih.gov/books/NBK258608/ [Accessed 8 February 2019]. 
4 Steingart KR, Henry M, Ng V et al. Fluorescence versus conventional sputum smear microscopy for tuberculosis: a systematic review. Lancet Infect Dis 2006;6:570-81.

5 Pfyffer GE, Wittwer F. Incubation time of mycobacterial cultures: How long is long enough to issue a final negative report to the clinician? J Clin Microbiol 2012;50:4188-9.

6 Nicol MP, Workman L, Isaacs W et al. Accuracy of the Xpert MTB/ RIF test for the diagnosis of pulmonary tuberculosis in children admitted to hospital in Cape Town, South Africa: A descriptive study. Lancet Infect Dis 2011;11:819-24.

7 Theron G, Peter J, van Zyl-Smit R et al. Evaluation of the Xpert MTB/RIF assay for the diagnosis of pulmonary tuberculosis in a high HIV prevalence setting. Am J Respir Crit Care Med 2011;184:132-40.

8 Sohn H, Aero AD, Menzies D et al. Xpert MTB/RIF testing in a low tuberculosis incidence, high-resource setting: Limitations in accuracy and clinical impact. Clin Infect Dis 2014;58:970-6.

9 Chakravorty S, Simmons AM, Rowneki M et al. The new Xpert MTB/ RIF Ultra: Improving detection of Mycobacterium tuberculosis and resistance to rifampin in an assay suitable for point-of-care testing. MBio 2017;8:e00812-7.

10 Dorman SE, Schumacher SG, Alland D et al. Xpert MTB/RIF Ultra for detection of Mycobacterium tuberculosis and rifampicin resistance: a prospective multicentre diagnostic accuracy study. Lancet Infect Dis 2018;18:76-84.

11 García-Basteiro AL, DiNardo A, Saavedra B et al. Point of care diagnostics for tuberculosis. Revista Portuguesa de Pneumologia (English Edition) 2018;24:73-85.

12 Witney AA, Cosgrove CA, Arnold A et al. Clinical use of whole genome sequencing for Mycobacterium tuberculosis. BMC Medicine 2016;14:46.

13 Satta G, Lipman M, Smith GP et al. Mycobacterium tuberculosis and whole-genome sequencing: how close are we to unleashing its full potential? Clin Microbiol Infect 2017;24:604-09.

14 Iseman MD, Wood AJJ (ed). Treatment of multidrug-resistant tuberculosis. N Engl J Med 1993;329:784-91.

15 Arnold A, Cooke GS, Kon OM et al. Drug-resistant TB: UK multicentre study (DRUMS): Treatment, management and outcomes in London and West Midlands 2008-2014. J Infect 2017;74:260-71.

16 Caminero JA, Scardigli A. Classification of antituberculosis drugs: A new proposal based on the most recent evidence. Eur Resp J 2015:46:887-93.

17 Diacon AH, Pym A, Grobusch M et al. The diarylquinoline TMC207 for multidrug-resistant tuberculosis. N Engl J Med 2009;360:2397405.

18 Diacon AH, Pym A, Grobusch MP et al. Multidrug-resistant tuberculosis and culture conversion with bedaquiline. N Engl J Med 2014:371:723-32
19 Gler MT, Skripconoka V, Sanchez-Garavito E et al. Delamanid for multidrug-resistant pulmonary tuberculosis. N Engl J Med 2012;366:2151-60.

20 Hafkin J, Hittel N, Martin A, Gupta R. Early outcomes in MDR-TB and XDR-TB patients treated with delamanid under compassionate use. Eur Respir ] 2017;50:1700311.

21 Gupta R, Gao M, Cirule A et al. Delamanid for extensively drugresistant tuberculosis. N Engl J Med 2015;373:291-2.

22 World Health Organization. WHO treatment guidelines for drugresistant tuberculosis: 2016 update. Geneva: WHO, 2016. https://apps. who.int/iris/bitstream/handle/10665/250125/9789241549639-eng. pdf? sequence $=1$ [Accessed 08 February 2019].

23 Falzon D, Schunemann HJ, Harausz E et al. World Health Organization treatment guidelines for drug-resistant tuberculosis, 2016 update. Eur Respir ] 2017;49:1602308.

24 Van Deun A, Maug AKJ, Salim MAH et al. Short, highly effective, and inexpensive standardized treatment of multidrug-resistant tuberculosis. Am J Respir Crit Care Med 2010;182:684-92.

25 Nunn AJ, Rusen ID, Van Deun A et al. Evaluation of a standardized treatment regimen of anti-tuberculosis drugs for patients with multi-drug-resistant tuberculosis (STREAM): Study protocol for a randomized controlled trial. Trials 2014;15:353.

26 Nathanson E, Gupta R, Huamani P et al. Adverse events in the treatment of multidrug-resistant tuberculosis: Results from the DOTS-Plus initiative. Int J Tuberc Lung Dis 2004;8:1382-4.

27 Bloss E, Kuksa L, Holtz TH et al. Adverse events related to multidrugresistant tuberculosis treatment, Latvia, 2000-2004. Int J Tuberc Lung Dis 2010;14:275-81

28 Tiberi S, du Plessis N, Walzl G et al. Tuberculosis: progress and advances in development of new drugs, treatment regimens, and host-directed therapies. Lancet Infect Dis 2018;18:e183-198.

29 Collaborative Group for the Meta-Analysis of Individual Patient Data in MDR-TB treatment-2017, Ahmad N, Ahuja SD et al. Treatment correlates of successful outcomes in pulmonary multidrug-resistant tuberculosis: an individual patient data metaanalysis. Lancet 2018;392:821-34.

30 World Health Organization. Rapid Communication: Key changes to treatment of multidrug- and rifampicin-resistant tuberculosis (MDR/ RR-TB). Geneva: WHO, 2018. www.who.int/tb/publications/2018/ WHO_RapidCommunicationMDRTB.pdf?ua=1 [Accessed 20 February 2019].

Address for correspondence: Prof Onn Min Kon, consultant respiratory physician and head of service for tuberculosis, Chest and Allergy Clinic, St Mary's Hospital, Imperial College Healthcare NHS Trust, Praed Street, London, W2 1NY, UK. Email: onn.kon@nhs.net 